\title{
Modelo de Clutter na modelagem de crescimento e produção de eucalipto em sistemas de integração lavoura-pecuária-floresta
}

\author{
Thiago Taglialegna Salles(1), Helio Garcia Leite(2), Sílvio Nolasco de Oliveira Neto(2), \\ Carlos Pedro Boechat Soares ${ }^{(2)}$, Haroldo Nogueira de Paiva(2) e Fabiano Lourenço dos Santos ${ }^{(3)}$
}

\begin{abstract}
(1)DAP Engenharia Florestal Ltda., Rua Papa João XXIII, no 9, Caixa Postal 50, Lourdes, CEP 36570-000 Viçosa, MG. E-mail: thiagotsalles@gmail.com (2)Universidade Federal de Viçosa, Departamento de Engenharia Florestal, CEP 36570-000 Viçosa, MG. E-mail: hgleite@gmail.com, snolasco@ufv.br, csoares@ufv.br, hnpaiva@ufv.br(3)Votorantim Siderurgia, Unidade Florestal, Caixa Postal 03, Zona Rural, CEP 38780-000 Vazante, MG. E-mail: fabiano.santos@vsiderurgia.com.br
\end{abstract}

Resumo - O objetivo deste trabalho foi definir a melhor forma de uso do modelo de Clutter para estimar o crescimento e a produção de clones de eucalipto em sistemas de integração lavoura-pecuária-floresta. Foram utilizados dados de sistemas de integração localizados na região noroeste do Estado de Minas Gerais. Os dados foram originados a partir de 180 parcelas permanentes de inventário florestal contínuo, de 30x40 m, contendo três fileiras de dez árvores, com amostragem casual estratificada de intensidade de uma parcela para cada 10 ha. O número médio de árvores por hectare foi de 242. Foram avaliadas plantas com idades de 20 a 95 meses. As melhores relações funcionais foram obtidas a partir do uso do modelo original de Clutter, com base na significância e nos sinais esperados das estimativas de seus parâmetros. O ajuste do modelo de Clutter deve ser feito na sua forma completa.

Termos para indexação: Eucalyptus, espaçamento, ILPF, índice de local, inventário florestal, sistemas agroflorestais.

\section{Clutter model in modeling growth and yield of eucalyptus in crop-livestock-forest integration systems}

\begin{abstract}
The objective of this work was to define the best form of use of the Clutter model, in order to estimate growth and yield of eucalyptus clones in crop-livestock-forest integration systems. Data were collected from integration systems located in the northwest region of the state of Minas Gerais, Brazil. The data were originated from 180 continuous inventory permanent plots of 30x40 m, with three ten-plant rows and stratified random sampling of one plot per $10 \mathrm{ha}$. The average number of trees per hectare was 242. Plants with ages from 20 to 95 months were evaluated. The best functional relationships were obtained using the original Clutter model, based on the significance and on the expected signs of its estimated parameters. The Clutter model should be adjusted on its unreduced form.
\end{abstract}

Index terms: Eucalyptus, spacing, ICLF, site index, forest inventory, crop forest integration.

\section{Introdução}

Nos últimos anos, principalmente com o crescente interesse por práticas agrícolas mais conservacionistas, os sistemas agroflorestais (SAF) têm sido estudados de forma crescente, em virtude de sua alta sustentabilidade (Santos \& Paiva, 2002; Mercer, 2004; Maia et al., 2006; Ayres \& Alfaia, 2007; Rodrigues et al., 2007; Aquino et al., 2008; Castro et al., 2009; Nair et al., 2009; Udawatta et al., 2010; Lima et al., 2011).

O sucesso da agrossilvicultura, especialmente com eucalipto, está fundamentado na adoção de planejamento criterioso, com base no levantamento minucioso de informações técnicas e econômicas
(Vale et al., 2004). Um dos principais entraves à adoção de sistemas mistos de cultivo é a alta demanda por capital. Sem incentivos, é provavel que, em regiões de lavouras produtivas, seja lenta a adoção destes sistemas, os quais dependem de preços relativos favoráveis e da disponibilidade de linhas de crédito que se adequem às necessidades do produtor. Mecanismos inovadores de financiamento são essenciais para promover e acelerar sua adoção em larga escala (Martha Junior et al., 2011). As avaliações econômicas são importantes para subsidiar os agentes de financiamento, os técnicos e os produtores envolvidos nesse tipo de investimento, e a análise da viabilidade desse tipo de projeto que está vinculada 
às estimativas de produtividade dos sistemas (Santos \& Paiva, 2002; Bentes-Gama, 2005).

Para a predição ou a projeção do crescimento e da produção de madeira, existem diferentes tipos de modelos matemáticos consagrados na literatura, entre os quais se destacam os do tipo povoamento (Campos \& Leite, 2009).

Contudo, os trabalhos com modelagem em SAF com eucalipto são escassos no Brasil. Dubè (1999), ao realizar estudos técnicos e econômicos em um SAF, utilizou um modelo simples, no qual apenas a idade foi considerada como variável independente para gerar estimativas de volume de madeira. Tsukamoto Filho (2003) obteve estimativas de volume satisfatórias em SAF a partir da aplicação do modelo mecanístico 3-PG. Souza (2005), ajustou um conjunto de modelos para prognose da produção de clones de eucalipto; entretanto, o modelo não conseguiu expressar volumes de madeira crescentes à medida que se aumentava a capacidade produtiva do local. Lopes (2007) adotou um modelo de distribuição diamétrica e obteve sucesso na sua aplicação. Em nenhum desses trabalhos, utilizou-se modelos de crescimento e produção (MCP) do tipo povoamento.

Os melhores representantes dos MCP do tipo povoamento são os modelos de densidade variável (expressa pela área basal, pelo diâmetro médio ou pela frequência), considerada como parte dinâmica do sistema de equações. Esses modelos são úteis para estimar o volume em unidade de área, principalmente para povoamentos submetidos ao desbaste.

O modelo desse tipo mais difundido no Brasil é o de Clutter (Campos \& Leite, 2009). Seu autor propôs um modelo de produção em volume, que foi ajustado a dados de parcelas estabelecidas em povoamentos de Pinus taeda L. e reduzido com base nas estatísticas do ajuste. No entanto, a função reduzida foi obtida a partir de condições diferentes daquelas em que é utilizada atualmente no país. O modelo na sua forma não reduzida apresenta, como componentes extras, termos que captam a interação entre suas variáveis duas a duas.

O objetivo deste trabalho foi definir a melhor forma de uso do modelo de Clutter para estimar o crescimento e a produção de clones de eucalipto em sistemas de integração lavoura-pecuária-floresta (ILPF).

\section{Material e Métodos}

Foram utilizados dados provenientes de sistemas ILPF sequenciais compostos por clones de híbridos de eucalipto (Eucalyptus camaldulensis Dehnh $\mathrm{x}$ E. grandis W. Hill, E. camaldulensis x E. urophylla S.T. Blake e E. camaldulensis $\mathrm{x}$ E. tereticornis Sm.), plantados no espaçamento de $10 \times 4 \mathrm{~m}$, com arroz, soja e braquiária (Urochloa brizantha) com pastejo de gado. Os plantios pertencem à unidade Aço-Florestal do grupo Votorantim, localizada nos municípios de Paracatu $\left(17^{\circ} 13^{\prime} 20^{\prime \prime} \mathrm{S}\right.$ e $\left.46^{\circ} 52^{\prime} 31^{\prime \prime W}\right)$, Vazante $\left(17^{\circ} 59^{\prime} 23^{\prime \prime S}\right.$ e $\left.46^{\circ} 53^{\prime} 59^{\prime \prime} \mathrm{W}\right)$ e

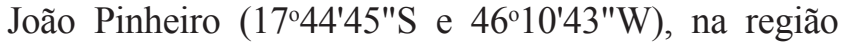
noroeste do Estado de Minas Gerais.

O consórcio foi feito com cultivo de arroz (Oryza sativa L., cultivar Guarani), no primeiro ano, e com soja [Glycine max (L.) Merr., cultivares Doko, Conquista e Vitória], no segundo. O preparo do solo consistiu, basicamente, de aração e gradagem antes do plantio de arroz e eucalipto, com a realização de calagem, tendo-se aplicado 4,0 $\mathrm{Mg} \mathrm{ha}^{-1}$ de calcário zincal 200 MMA (PRNT 85\%). A adubação mineral para o plantio de arroz consistiu de $200 \mathrm{~kg} \mathrm{ha}^{-1}$ de N-P-K (5-25-15). Para o eucalipto, foram aplicados 150 g por cova de N-P-K (10-28-6 ou 6-30-6). Antes do plantio da soja, foram realizadas uma gradagem aradora e duas gradagens niveladoras. Nessa ocasião, foram incorporados ao solo mais $3 \mathrm{Mg} \mathrm{ha}^{-1}$ de calcário zincal 200 MMA (PRNT 85\%). O espaçamento de plantio entre as linhas foi de $0,45 \mathrm{~m}$ para o arroz e a soja, tendo-se utilizado 80 e 24 sementes por metro linear, respectivamente. Para evitar competição, respeitou-se o espaçamento mínimo de $1 \mathrm{~m}$ entre as linhas de plantio de eucalipto e as linhas de arroz e de soja. No segundo ano, foi feita a primeira manutenção do povoamento de eucalipto, que consistiu em capinas manuais, conservação de estradas e aceiros, e o combate a formigas com formicida granulado Atamex, na dosagem de 2,0 $\mathrm{kg} \mathrm{ha}^{-1}$.

A partir do ano seguinte, houve a formação de pastagens de braquiária manejadas para engorda de gado de corte. Foi respeitado espaçamento mínimo de 1,0 m entre as linhas de plantio de eucalipto e a área de semeadura da gramínea. A quantidade de sementes foi de aproximadamente $10,0 \mathrm{~kg} \mathrm{ha}^{-1}$. As sementes foram misturadas com fosfato natural (400 $\left.\mathrm{kg} \mathrm{ha}^{-1}\right) \mathrm{e}$ superfosfato simples (100 kg ha-1). Para a calagem, 1,0 $\mathrm{Mg} \mathrm{ha}^{-1}$ de calcário dolomítico zincal $200 \mathrm{MMA}$ (PRNT 85\%) foi incorporado ao solo. O preparo de solo necessário para a semeadura consistiu em gradagem pesada à profundidade de $20,0 \mathrm{~cm}$, seguida de gradagem niveladora. Nesse mesmo ano, os eucaliptos foram desramados até a altura de 4,0 $\mathrm{m}$ do solo. 
No quarto ano, iniciou-se o período de utilização da pastagem para engorda de bois. As unidades de manejo para o gado foram compostas por piquetes de 37,5 ha cada um, dispostos lado a lado em áreas contíguas, com capacidade de suporte estimada em 1,0 UA ha- ${ }^{-1}$ no inverno e 2,0 UA ha ${ }^{-1}$ no verão. Houve manutenção (conservação de estradas e aceiros e combate a formigas) dos povoamentos de eucalipto, com uma segunda desrama feita até $6 \mathrm{~m}$ de altura.

No quinto ano, houve manutenção apenas do componente florestal. A partir do sexto ano, estabeleceuse o sistema silvipastoril com periodicidade bianual de vendas de bois gordos (Dubè, 1999; Tsukamoto Filho, 2003).

Os primeiros sistemas foram implantados em 1993, e a coleta de dados foi realizada em cinco ocasiões, uma vez por ano, de 2004 a 2008. Dos 38 clones presentes nos ILPF, os sete mais bem amostrados foram selecionados para análise de modelagem (Tabela 1).

Os dados foram originados a partir de 180 parcelas permanentes de inventário florestal contínuo, de 30x40 m $\left(1.200 \mathrm{~m}^{2}\right)$, contendo três fileiras de dez árvores (30 árvores por parcela), implantadas de acordo com os critérios de inventário florestal contínuo por amostragem casual estratificada, com intensidade de 1:10 (uma parcela para cada 10 ha). Cada parcela consistia apenas de um clone plantado. Não foram lançadas parcelas em talhões com área inferior a 15 ha. As idades observadas foram de 20 a 95 meses, e o número médio de árvores por hectare foi igual a 242 . Em todas as parcelas mensuradas e em todos os anos, foram avaliados: altura total $(\mathrm{Ht})$, diâmetro à altura do peito (DAP) e idade do povoamento (I). Também foram determinados para cada parcela: área basal (B), volume comercial (diâmetro mínimo igual a $4,0 \mathrm{~cm}$ ) com casca (V) e altura total das árvores dominantes (Hd). A área basal e o volume foram extrapolados para hectare. Os dados foram submetidos à análise de consistência, tendo-se eliminado os "outliers" e os dados de parcelas com menos de três medições.

Estimou-se o índice de local (S), representativo da capacidade produtiva do lugar, por ser considerado variável independente do modelo aplicado. Para tanto, utilizou-se o método da curva-guia, conforme Oliveira et al. (2008), que geralmente é usado para a classificação da capacidade produtiva.

Dois modelos (Logístico e Gompertz) foram ajustados a cada clone, para estimar a altura dominante. A escolha entre os modelos foi feita com base no coeficiente de correlação $\left(\mathrm{r}_{\hat{y} y}\right)$ e na análise dos comportamentos gráficos das curvas ajustadas. Posteriormente, os modelos foram transformados para estimar os índices de local, de acordo com Campos \& Leite (2009), para uma idade-índice igual a 72 meses. As relações funcionais resultantes dos modelos Logístico e Gompertz foram:

$\mathrm{S}=\mathrm{Hd}\left[1+\beta_{1} \exp \left(-\beta_{2} \mathrm{I}\right)\right]\left[1+\beta_{1} \exp \left(-\beta_{2} \mathrm{I}_{\mathrm{I}}\right)\right]^{-1}+\varepsilon \mathrm{e}$

$S=H d\left\{\exp \left[-\exp \left(\beta_{1}-\beta_{2} I_{I}\right)\right]\right\}\left\{\exp \left[-\exp \left(\beta_{1}-\beta_{2} I\right)\right]\right\}^{-1}+\varepsilon$,

em que: Hd é a altura dominante, em metros; S, é o índice de local; I, é a idade, em meses; I I , é a idade-índice, em meses; $\beta_{\mathrm{i}}$, são os parâmetros do modelo; e $\varepsilon$, é o erro aleatório, em que $\varepsilon \sim \mathrm{N}\left(0, \sigma^{2}\right)$.

Para a projeção do crescimento e da produção, utilizou-se o modelo completo de Clutter (1963):

$\ln \mathrm{B}_{2}=\ln \left(\mathrm{B}_{1}\right)\left(\mathrm{I}_{1} \mathrm{I}_{2}{ }^{-1}\right)+\alpha_{0}\left(1-\mathrm{I}_{1} \mathrm{I}_{2}^{-1}\right)+\alpha_{1}\left(1-\mathrm{I}_{1} \mathrm{I}_{2}^{-1}\right) \mathrm{S}_{1}+\varepsilon \mathrm{e}$ $\ln V_{2}=\beta_{0}+\beta_{1} I_{2}^{-1}+\beta_{2} S_{1}+\beta_{3} \ln \left(B_{2}\right)+\beta_{4} S_{1} I_{2}^{-1}+$

$\beta_{5} S_{1} \ln \left(B_{2}\right)+\beta_{6} \ln \left(B_{2}\right) I_{2}^{-1}+\varepsilon$,

em que: $\mathrm{I}_{1}$ é a idade presente, em meses; $\mathrm{I}_{2}$, é a idade futura, em meses; $\mathrm{B}_{1}$, é a área basal na idade $\mathrm{I}_{1}$, em $\mathrm{m}^{2} \mathrm{ha}^{-1} ; \mathrm{B}_{2}$, é a área basal na idade $\mathrm{I}_{2}, \mathrm{em} \mathrm{m}^{2} \mathrm{ha}^{-1}$;

Tabela 1. Amplitude e valores médios de idade, altura total (Ht), diâmetro à altura do peito (DAP), área basal (B) e volume com casca (V) de sete clones de eucalipto em sistemas de integração lavoura-pecuária-floresta.

\begin{tabular}{|c|c|c|c|c|c|c|c|c|c|c|c|c|c|c|}
\hline \multirow{2}{*}{ Clone } & \multicolumn{2}{|c|}{ Idade (meses) } & \multicolumn{3}{|c|}{$\mathrm{Ht}(\mathrm{m})$} & \multicolumn{3}{|c|}{ DAP $(\mathrm{cm})$} & \multicolumn{3}{|c|}{$\mathrm{B}\left(\mathrm{m}^{2} \mathrm{ha}^{-1}\right)$} & \multicolumn{3}{|c|}{$\mathrm{V}\left(\mathrm{m}^{3} \mathrm{ha}^{-1}\right)$} \\
\hline & Mín. & Máx. & Mín. & Méd. & Máx. & Mín. & Méd. & Máx. & Mín. & Méd. & Máx. & Mín. & Méd. & Máx. \\
\hline 01 & 31 & 82 & 6,7 & 22,3 & 30,7 & 6,1 & 19,5 & 27,6 & 3,8 & 7,9 & 12,5 & 20,8 & 85,0 & 169,7 \\
\hline 02 & 21 & 71 & 5,7 & 19,3 & 29,5 & 6,0 & 17,6 & 33,0 & 2,2 & 6,0 & 9,3 & 10,0 & 52,6 & 101,0 \\
\hline 03 & 31 & 82 & 8,6 & 21,5 & 32,1 & 6,3 & 18,3 & 27,9 & 2,9 & 6,8 & 10,7 & 16,7 & 72,8 & 150,9 \\
\hline 04 & 21 & 86 & 6,3 & 22,2 & 33,4 & 6,9 & 18,2 & 25,9 & 2,3 & 6,9 & 10,9 & 9,2 & 72,6 & 149,1 \\
\hline 05 & 20 & 82 & 5,3 & 20,9 & 33,1 & 6,1 & 19,0 & 30,1 & 1,1 & 7,1 & 12,3 & 3,4 & 70,0 & 175,1 \\
\hline 06 & 20 & 94 & 2,2 & 20,0 & 37,9 & 4,5 & 19,8 & 35,9 & 1,6 & 8,1 & 15,7 & 6,1 & 73,2 & 191,1 \\
\hline 07 & 29 & 94 & 8,5 & 23,5 & 34,6 & 6,4 & 20,7 & 32,1 & 2,8 & 8,8 & 14,3 & 14,8 & 91,0 & 195,6 \\
\hline Geral & 20 & 94 & 2,2 & 20,7 & 37,9 & 4,5 & 19,4 & 35,9 & 1,1 & 7,8 & 15,7 & 3,4 & 72,9 & 195,6 \\
\hline
\end{tabular}


$\mathrm{S}_{1}$, é o índice de local na idade $\mathrm{I}_{1} ; \mathrm{V}_{2}$, é o volume na idade $\mathrm{I}_{2}$ em m $\mathrm{m}^{3} \mathrm{ha}^{-1} ; \alpha_{\mathrm{i}}$ e $\beta_{\mathrm{i}}$, são os parâmetros do modelo; e $\varepsilon$ é o erro aleatório, em que $\varepsilon \sim \mathrm{N}\left(0, \sigma^{2}\right)$. Por se tratar de sistema de equações simultâneas, a estimação dos parâmetros foi feita pelo método de mínimos quadrados, em dois estágios.

Para obter uma forma funcional específica para estimar o crescimento e a produção de cada um dos clones, as equações foram reduzidas de acordo com o sinal esperado dos parâmetros e das suas respectivas significâncias obtidas nos ajustes do modelo. O sinal esperado para os coeficientes $\beta_{1}$ e $\beta_{4}$ foi negativo, e para os demais positivo. Neste processo, buscou-se a construção de modelos biologicamente consistentes, tendo-se mantido, em sua forma final, as variáveis: área basal futura $\left(\mathrm{B}_{2}\right)$, índice de local $\left(\mathrm{S}_{1}\right)$ e idade futura $\left(\mathrm{I}_{2}\right)$, nas suas formas isoladas ou nas suas interações.

É importante ressaltar que, ao utilizar o método dos mínimos quadrados em dois estágios, os testes estatísticos convencionais $\mathrm{F}$ e $\mathrm{t}$ não são estritamente aplicáveis, e que o coeficiente de determinação $\left(\mathrm{R}_{\hat{y} \mathrm{y}}^{2}\right)$ deve ser visto com cautela (Debertin, 1975). No presente trabalho, para avaliar a significância dos parâmetros utilizados, foi verificado se era verdadeira a hipótese de o valor absoluto de cada parâmetro ser maior que o dobro do seu respectivo erro-padrão. Em caso afirmativo, a sua estimativa foi considerada confiável, a 5\% de probabilidade. Esse método baseiase na estatística t de Student, que é dada pela razão entre o valor estimado do parâmetro e o seu erro-padrão. Uma vez que o $t$ tabelado a $5 \%$ de probabilidade tende a valores próximos, mas menores que 2,0, para um grande número de observações, um parâmetro sempre será significativo quando for maior do que o dobro de seu erro-padrão.

Para avaliar o desempenho dos modelos em projetar o volume e a área basal, foram calculadas as estatísticas: raiz quadrada do erro-médio (RQEM) e erro-médio percentual de predição (bias\%), conforme Siipilehto \& Siitonen (2004) e Siipilehto et al. (2007):

$$
\begin{aligned}
& \text { bias\% }=100 \frac{1}{\mathrm{n}} \sum_{\mathrm{i}=1}^{\mathrm{n}}\left[\left(\mathrm{Y}_{\mathrm{i}}-\hat{\mathrm{Y}}_{\mathrm{i}}\right) \mathrm{Y}_{\mathrm{i}}\right] \mathrm{e} \\
& \text { RQEM }=\sqrt{\frac{\sum_{\mathrm{i}=1}^{\mathrm{n}}\left(\mathrm{Y}_{\mathrm{i}}-\hat{\mathrm{Y}}_{\mathrm{i}}\right)^{2}}{\mathrm{n}-1}},
\end{aligned}
$$

em que: $Y_{i}$ e $\hat{Y}_{i}$ são o valor observado e estimado da variável sob análise, respectivamente; e n é o número de casos.

Para melhor interpretar a dispersão dos valores de área basal e volume, em comparação às suas estimativas, foram gerados gráficos para relacionar essas variáveis por clone, tendo-se considerado três classes de produtividade, definidas empiricamente por classes I, II e III.

Para permitir comparações entre as produtividades médias em área basal e volume entre os clones, foram gerados gráficos a partir das equações de área basal futura $\left(\mathrm{B}_{2}\right)$ e volume futuro $\left(\mathrm{V}_{2}\right)$.

\section{Resultados e Discussão}

Com base no coeficiente de correlação $\left(r_{\hat{y} y}\right)$, houve bom desempenho no ajuste dos modelos às alturas dominantes dos eucaliptos dos sistemas ILPF (Tabela 2), como também observado por Demolinari et al. (2007) e Soares et al. (2009). As curvas geradas pelos modelos descreveram bem o comportamento de $\mathrm{Hd}$ em relação à idade, tendo passado pelos pontos correspondentes aos dados observados. Assim, a partir dos coeficientes $\beta_{1}$ e $\beta_{2}$ obtidos, foi possível estimar valores de $\mathrm{S}$ consistentes, ao se aplicar as relações funcionais transformadas para obtenção dos índices de local.

As estimativas dos parâmetros, que apresentaram valores absolutos maiores do que o dobro de seus respectivos erros-padrão e sinal conforme o esperado, foram consideradas significativas a $5 \%$ de probabilidade (Tabela 3). Os ajustes finais foram feitos apenas a partir desses parâmetros e originaram as equações de projeção.

Os ajustes resultaram em relações funcionais diferentes para cada clone (Tabela 4). Caso a forma reduzida do modelo de Clutter fosse aplicada diretamente, haveria

Tabela 2. Modelos atribuídos a cada clone de eucalipto, para classificação de índice de local em sistemas de integração lavoura-pecuária-floresta, e respectivos coeficientes estimados $\left(\hat{\beta}_{\mathrm{i}}\right)$ e coeficiente de correlação $\left(\mathrm{r}_{\hat{y} \mathrm{y}}\right)$.

\begin{tabular}{lcrrc}
\hline Modelo & Clone & \multicolumn{1}{c}{$\hat{\beta}_{1}$} & $\hat{\beta}_{2}$ & $\mathrm{r}_{\hat{y} y}$ \\
\hline \multirow{3}{*}{ Logístico } & 02 & 9,5545 & 0,0864 & 0,88 \\
& 03 & 10,6567 & 0,0703 & 0,96 \\
& 07 & 4,7198 & 0,0486 & 0,97 \\
\hline \multirow{3}{*}{ Gompertz } & 01 & 1,1943 & 0,0517 & 0,96 \\
& 04 & 1,1052 & 0,0455 & 0,97 \\
& 05 & 0,8315 & 0,0270 & 0,94 \\
\hline
\end{tabular}


ajuste satisfatório apenas aos clones 05 e 06 . Isso indica que o modelo em sua forma completa é mais flexível, e que as interações entre as suas variáveis nem sempre são não significativas, conforme observado no trabalho original de Clutter (1963). Oliveira et al. (2009a) também obtiveram melhor resultado ao utilizar equação de projeção de volume diferente do modelo diretamente reduzido.

Houve bom ajuste a todos os materiais genéticos modelados. O bias\% computado para as equações mostrou-se satisfatório, tendo ficado abaixo de $1 \%$ em todos os casos (Tabela 4). Dias et al. (2005), ao utilizar modelo de crescimento e produção em povoamentos desbastados de eucalipto, relataram que estimativas com valores de bias\% menores que $10 \%$ são consideradas precisas. A RQEM, que representa a raiz quadrada do erro-médio das estimativas, deve estar o mais próximo possível de 0 (Behling, 2009). Em relação a essa estatística, as estimativas menos exatas foram as feitas para o clone 02 , com valores de 0,10 , para o logaritmo da área basal, e de 0,15 para o logaritmo do volume. Valores semelhantes foram obtidos por Zonete et al. (2010), ao estimar o volume de eucalipto.

Observou-se que as curvas de volume e área basal geradas pelo modelo descreveram com fidelidade as tendências de crescimento dos clones de eucalipto nos sistemas ILPF, pois passaram pelos pontos que representam os dados observados em campo (Figura 1). As três classes de produtividade foram bem representadas em todos os clones, uma vez que o feixe de curvas compreendeu a amplitude dos dados obtidos em todos os casos.

O clone 02 obteve o pior desempenho na análise da produtividade média em área basal e volume (Figura 2).

Tabela 3. Coeficientes estimados para os parâmetros dos modelos de projeção de volume e de área basal de sete clones de eucalipto em sistemas de integração lavoura-pecuária-floresta e, entre parênteses, medidas de seus erros-padrão ${ }^{(1)}$.

\begin{tabular}{lccccccccc}
\hline Clone & $\hat{\alpha}_{0}$ & $\hat{\alpha}_{1}$ & $\hat{\beta}_{0}$ & $\hat{\beta}_{1}$ & $\hat{\beta}_{2}$ & $\hat{\beta}_{3}$ & $\hat{\beta}_{4}$ & $\hat{\beta}_{5}$ & $\hat{\beta}_{6}$ \\
\hline \multirow{2}{*}{01} & 2,9968 & - & 4,1183 & $-50,7283$ & - & - & - & 0,0220 \\
& $(0,057)$ & - & $(0,359)$ & $(8,417)$ & - & - & - & $(0,004)$ \\
\hline \multirow{2}{*}{02} & - & 0,1013 & 3,2841 & $-138,1577$ & 0,0353 & - & - & - \\
& - & $(0,002)$ & $(0,205)$ & $(10,837)$ & $(0,006)$ & - & - & - & $(10,204)$ \\
\hline \multirow{2}{*}{03} & 2,8097 & - & 0,0289 & - & - & - & $-2,7891$ & 2,9705 & 33,9014 \\
& $(0,055)$ & - & $(0,002)$ & - & - & - & $(0,462)$ & $(0,138)$ & $(10,498)$ \\
\hline \multirow{2}{*}{04} & 2,8204 & - & 4,3114 & $-60,5730$ & - & - & - & 0,0191 & - \\
& $(0,044)$ & - & $(0,203)$ & $(4,061)$ & - & - & - & $(0,002)$ & - \\
\hline \multirow{2}{*}{05} & 1,9496 & 0,0355 & 2,8240 & $-72,7828$ & 0,0485 & 0,6821 & - & - & - \\
& $(0,406)$ & $(0,014)$ & $(0,425)$ & $(10,739)$ & $(0,008)$ & $(0,155)$ & - & - \\
\hline \multirow{2}{*}{06} & 2,3180 & 0,0220 & 1,7205 & $-22,4677$ & 0,0103 & 1,2758 & - & - \\
& $(0,148)$ & $(0,005)$ & $(0,119)$ & $(2,222)$ & $(0,002)$ & $(0,052)$ & - & - \\
07 & 2,9927 & - & 3,1124 & - & - & - & $-2,7501$ & 0,0284 & 25,1953 \\
& $(0,031)$ & - & $(0,148)$ & - & - & - & $(0,267)$ & $(0,001)$ & $(6,100)$ \\
\hline
\end{tabular}

${ }^{(1)}$ As estimativas representadas por hífen apresentaram valor não significativo ou sinal inconsistente, tendo-se retirado o respectivo parâmetro do modelo.

Tabela 4. Modelos para projeção de volume e de área basal atribuídos a sete clones de eucalipto em sistemas de integração lavoura-pecuária-floresta, medidas de erro-médio percentual de predição (bias\%) e raiz quadrada do erro-médio (RQEM).

\begin{tabular}{|c|c|c|c|c|c|c|}
\hline Clone & Equações volume & bias $(\%)$ & RQEM & Equações área basal & bias $(\%)$ & RQEM \\
\hline 01 & $\ln B_{2}=\ln \left(B_{1}\right)\left(I_{1} I_{2}^{-1}\right)+\alpha_{0}\left(1-I_{1} I_{2}^{-1}\right)$ & 0,20 & 0,05 & $\ln V_{2}=\beta_{0}+\beta_{1} I_{2}^{-1}+\beta_{5} S_{1} \ln \left(B_{2}\right)$ & 0,04 & 0,09 \\
\hline 02 & $\ln B_{2}=\ln \left(B_{1}\right)\left(I_{1} I_{2}^{-1}\right)+\alpha_{1}\left(1-I_{1} I_{2}^{-1}\right) S_{1}$ & 0,93 & 0,10 & $\ln V_{2}=\beta_{0}+\beta_{1} I_{2}^{-1}+\beta_{2} S_{1}+\beta_{6} \ln \left(B_{2}\right) I_{2}^{-1}$ & 0,32 & 0,15 \\
\hline 03 & $\ln B_{2}=\ln \left(B_{1}\right)\left(I_{1} I_{2}^{-1}\right)+\alpha_{0}\left(1-I_{1} I_{2}^{-1}\right)$ & 0,19 & 0,05 & $\ln V_{2}=\beta_{0}+\beta_{4} S_{1} I_{2}^{-1}+\beta_{5} S_{1} \ln \left(B_{2}\right)+\beta_{6} \ln \left(B_{2}\right) I_{2}^{-1}$ & 0,16 & 0,06 \\
\hline 04 & $\ln B_{2}=\ln \left(B_{1}\right)\left(I_{1} I_{2}^{-1}\right)+\alpha_{0}\left(1-I_{1} I_{2}^{-1}\right)$ & 0,15 & 0,07 & $\ln V_{2}=\beta_{0}+\beta_{1} I_{2}^{-1}+\beta_{5} S_{1} \ln \left(B_{2}\right)$ & 0,01 & 0,08 \\
\hline 05 & $\ln B_{2}=\ln \left(B_{1}\right)\left(I_{1} I_{2}^{-1}\right)+\alpha_{0}\left(1-\mathrm{I}_{1} I_{2}^{-1}\right)+\alpha_{1}\left(1-\mathrm{I}_{1} \mathrm{I}_{2}^{-1}\right) \mathrm{S}_{1}$ & 0,30 & 0,07 & $\ln V_{2}=\beta_{0}+\beta_{1} I_{2}^{-1}+\beta_{2} S_{1}+\beta_{3} \ln \left(B_{2}\right)$ & 0,06 & 0,13 \\
\hline 06 & $\ln B_{2}=\ln \left(B_{1}\right)\left(I_{1} I_{2}^{-1}\right)+\alpha_{0}\left(1-I_{1} I_{2}^{-1}\right)+\alpha_{1}\left(1-I_{1} I_{2}^{-1}\right) S_{1}$ & 0,17 & 0,06 & $\ln V_{2}=\beta_{0}+\beta_{1} I_{2}^{-1}+\beta_{2} S_{1}+\beta_{3} \ln \left(B_{2}\right)$ & 0,11 & 0,10 \\
\hline 07 & $\ln B_{2}=\ln \left(B_{1}\right)\left(I_{1} I_{2}^{-1}\right)+\alpha_{0}\left(1-I_{1} I_{2}^{-1}\right)$ & 0,30 & 0,06 & $\ln V_{2}=\beta_{0}+\beta_{4} S_{1} I_{2}^{-1}+\beta_{5} S_{1} \ln \left(B_{2}\right)+\beta_{6} \ln \left(B_{2}\right) I_{2}^{-1}$ & 0,19 & 0,07 \\
\hline
\end{tabular}


O melhor material genético, quanto ao crescimento em área basal, foi o de número 06 , e ao volume, o de número 01, seguido pelos clones 06 e 04 .

Ao adotar o mesmo sistema ILPF avaliado no presente trabalho, Dubè (1999) utilizou um modelo exponencial para determinar o volume por hectare do componente florestal, tendo observado, aos sete anos, cerca de 150,0 $\mathrm{m}^{3} \mathrm{ha}^{-1}$. Tsukamoto Filho (2003), ao avaliar o mesmo sistema ILPF, fez uso do modelo 3-PG e encontrou volume maior (em torno de $180,0 \mathrm{~m}^{3} \mathrm{ha}^{-1}$ aos sete anos) do que o estimado por Dubè (1999).
Esses valores, quando comparados aos volumes médios obtidos pelas equações utilizadas no presente trabalho (Figura 2), cujos valores variaram de 82,5 a $143,0 \mathrm{~m}^{3}$ $\mathrm{ha}^{-1}$ aos sete anos, foram considerados superestimados.

A modelagem é utilizada como suporte à decisão na implantação e no manejo de espécies florestais. Portanto, superestimar ou subestimar o volume de madeira de um empreendimento pode comprometer as tomadas de decisão.Além disso, o uso de modelos mais simples que, ao contrário do de Clutter, não consideram a variável índice de local (S), pode gerar idades técnicas de corte


Idade (meses)


Idade (meses)

$-\mathrm{S}=\mathrm{I} \quad \mathrm{S}=\mathrm{II} \quad \ldots-\cdots \mathrm{S}=\mathrm{III}$

Figura 1. Área basal e volume estimados para capacidade produtiva alta (I), média (II) e baixa (III) de sete clones de eucalipto amostrados em sistemas de integração lavoura-pecuária-floresta. 
(ITC) iguais para locais com classe de produtividade diferentes. A aplicação do fator de forma, como em Oliveira et al. (2009b), muitas vezes decorrente da escassez de dados temporais, também pode apresentar estimativas de menor qualidade do que as obtidas por modelagem (Rolim et al., 2006). O procedimento mais indicado seria o realizado por Souza et al. (2007), que, ao avaliar a viabilidade econômica de um sistema agroflorestal, fizeram uso de um MCP para obter os dados de volume de madeira necessários para a avaliação.
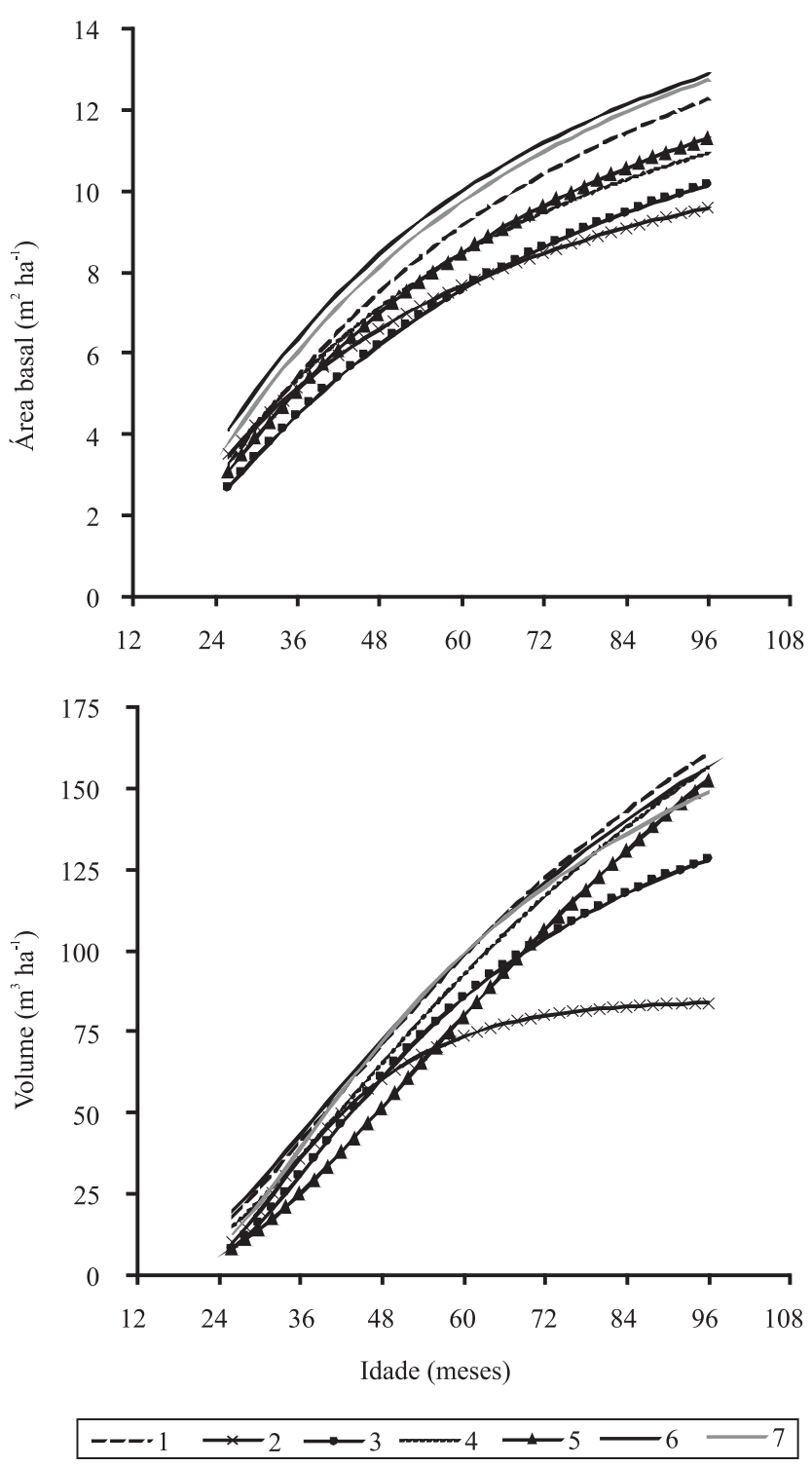

Figura 2. Crescimento e produtividade média em área basal e volume de sete clones de eucalipto amostrados em sistemas de integração lavoura-pecuária-floresta.
Em determinadas situações, há dificuldades no ajuste do modelo de Clutter na sua forma reduzida (sem as interações entre as variáveis), em decorrência das características dos dados disponíveis. Nesses casos, às vezes, são ajustados modelos volumétricos mais simples, com um número menor de variáveis independentes (Campos \& Leite, 2009). O modelo de Clutter na sua forma não reduzida se ajustou normalmente a todos os clones avaliados e foi eficiente para projetar as suas áreas basais e os seus volumes dentro do ILPF. Assim, deve ser dada preferência ao seu uso, já que, além de assumir a forma usual (reduzida), quando esta corresponde à tendência do estrato, o modelo pode assumir outras formas finais compatíveis com diferentes tendências de crescimento, como observado para os clones 02 e 05 (Figura 1).

\section{Conclusão}

O modelo de Clutter deve ser ajustado, inicialmente, na sua forma completa, e a sua melhor forma de uso deve ser definida a partir da significância e do sinal das estimativas dos seus parâmetros.

\section{Agradecimentos}

Ao Conselho Nacional de Desenvolvimento Científico e Tecnológico, pelo apoio financeiro; e à Votorantim Metais, pelo fornecimento dos dados para a realização deste trabalho.

\section{Referências}

AQUINO, A.M. de; MELOVIRGÍNIO FILHO, E. de; RICCI, M. dos S.F.; CASANOVES, F. Populações de minhocas em sistemas agroflorestais com café convencional e orgânico. Ciência e Agrotecnologia, v.32, p.1184-1188, 2008.

AYRES, M.I. da C.; ALFAIA, S.S. Calagem e adubação potássica na produção do cupuaçuzeiro em sistemas agroflorestais da Amazônia Ocidental. Pesquisa Agropecuária Brasileira, v.42, p.957-963, 2007.

BEHLING, M. Nutrição, partição de biomassa e crescimento de povoamentos de teca em Tangará da Serra-MT. 2009. 156p. Tese (Doutorado) - Universidade Federal de Viçosa, Viçosa.

BENTES-GAMA, M. de M.; SILVA, M.L. da; MONTOYA VILCAHUAMÁN, L.J.; LOCATELLI, M. Análise econômica de sistemas agroflorestais na Amazônia Ocidental, Machadinho D’Oeste-RO. Revista Árvore, v.29, p.401-411, 2005.

CABANETTES, A.; AUCLAIR, D.; IMAM, W. Diameter and height growth curves for widely-spaced trees in European agroforestry. Agroforestry Systems, v.43, p.169-181, 1999. 
CAMPOS, J.C.C.; LEITE, H.G. Mensuração florestal: perguntas e respostas. 3.ed. Viçosa: UFV, 2009. 548p.

CASTRO, A.P. de; FRAXE, T. de J.P.; SANTIAGO, J.L.; MATOS, R.B.; PINTO, I.C. Os sistemas agroflorestais como alternativa de sustentabilidade em ecossistemas de várzea no Amazonas. Acta Amazonica, v.39, p.279-288, 2009.

CLUTTER, J.L. Compatible growth and yield models for loblolly pine. Forest Science, v.9, p.354-371, 1963.

DEBERTIN, D.L. Significance tests of regression coefficients: an additional reminder. American Journal of Agricultural Economics, v.57, p.124, 1975.

DEMOLINARI, R. de A.; SOARES, C.P.B.; LEITE, H.G.; SOUZA, A.L. de. Crescimento de plantios clonais de eucalipto não desbastados na região de Monte Dourado (PA). Revista Árvore, v.31, p.503-512, 2007.

DIAS, A.N.; LEITE, H.G.; CAMPOS, J.C.C.; COUTO, L.; CARVALHO, A.F. de. Emprego de um modelo de crescimento e produção em povoamentos desbastados de eucalipto. Revista Árvore, v.29, p.731-739, 2005.

DUBÈ, F. Estudos técnicos e econômicos de sistemas agroflorestais com Eucalyptus sp. no nordeste do Estado de Minas Gerais: o caso da Companhia Mineira de Metais. 1999. 146p. Dissertação (Mestrado) - Universidade Federal de Viçosa, Viçosa.

LIMA, S.S. de; LEITE, L.F.C.; OLIVEIRA, F. das C.; COSTA, D.B. da. Atributos químicos e estoques de carbono e nitrogênio em Argissolo Vermelho-Amarelo sob sistemas agroflorestais e agricultura de corte e queima no norte do Piauí. Revista Árvore, v.35, p.51-60, 2011.

LOPES, P.F. Modelo de distribuição de diâmetros para clones de eucalipto em sistema agroflorestal. 2007. 32p. Dissertação (Mestrado) - Universidade Federal de Viçosa, Viçosa.

MAIA, S.M.F.; XAVIER, F.A. da S.; OLIVEIRA, T.S. de; MENDONÇA, E. de S.; ARAÚJO FILHO, J.A. de. Impactos de sistemas agroflorestais e convencional sobre a qualidade do solo no semi-árido cearense. Revista Árvore, v.30, p.837-848, 2006.

MARTHA JUNIOR, G.B.; ALVES, E.R. de A.; CONTINI, E. Dimensão econômica de sistemas de integração lavourapecuária. Pesquisa Agropecuária Brasileira, v.46, p.11171126, 2011.

MERCER, D.E. Adoption of agroforestry innovations in the tropics: a review. Agroforestry Systems, v.61, p.311-328, 2004.

NAIR, P.K.R.; KUMAR, B.M.; NAIR, V.D. Agroforestry as a strategy for carbon sequestration. Journal of Plant Nutrition and Soil Science, v.172, p.10-23, 2009.

OLIVEIRA， M.L.R. de; LEITE， H.G.; NOGUEIRA， G.S.; CAMPOS, J.C.C. Modelagem e prognose em povoamentos não desbastados de clones de eucalipto. Revista Árvore, v.33, p.841-852, 2009a.

OLIVEIRA, M.L.R. de; LEITE, H.G.; NOGUEIRA, G.S.; GARCIA, S.L.R.; SOUZA, A.L. de. Classificação da capacidade produtiva de povoamentos não desbastados de clones de eucalipto. Pesquisa Agropecuária Brasileira, v.43, p.1559-1567, 2008.

OLIVEIRA, T.K. de; MACEDO, R.L.G.; VENTURIN, N.; HIGASHIKAWA, E.M.; Desempenho silvicultural e produtivo de eucalipto sob diferentes arranjos espaciais em sistema agrossilvipastoril. Pesquisa Florestal Brasileira, n.60, p.1-9, 2009b. Número especial.

RODRIGUES, E.R.; CULLEN JUNIOR, L.; BELTRAME, T.P.; MOSCOGLIATO, A.V.; SILVA, I.C. da. Avaliação econômica de sistemas agroflorestais implantados para recuperação de reserva legal no Pontal do Paranapanema, São Paulo. Revista Árvore, v.31, p.941-948, 2007

ROLIM, S.G.; COUTO, H.T.Z. do; JESUS, R.M. de; FRANÇA, J.T. Modelos volumétricos para a Floresta Nacional do Tapirapé-Aquirí, Serra dos Carajás (PA). Acta Amazonica, v.36, p.107-114, 2006.

SANTOS, M.J.C. dos; PAIVA, S.N. de. Os sistemas agroflorestais como alternativa econômica em pequenas propriedades rurais: estudo de caso. Ciência Florestal, v.12, p.135-141, 2002.

SIIPILEHTO, J.; SARKKOLA, S.; MEHTÄTALO, L. Comparing regression estimation techniques when predicting diameter distributions of scots pine on drained peatlands. Silva Fennica, v.41, p.333-349, 2007.

SIIPILEHTO, J.; SIITONEN, J. Degree of previous cutting in explaining the differences in diameter distributions between mature managed and natural Norway spruce forests. Silva Fennica, v.38, p.425-435, 2004.

SOARES, C.P.B.; DEMOLINARI, R. de A.; LEITE, H.G.; SOUZA, A.L. de. Predição do crescimento em povoamentos clonais de eucalipto não desbastados utilizando matrizes de transição não estacionárias. Revista Árvore, v.33, p.831-840, 2009.

SOUZA, A.N. Crescimento, produção e análise econômica de povoamentos de Eucalyptus sp. em sistemas agroflorestais. 2005. 203p. Tese (Doutorado) - Universidade Federal de Lavras, Lavras.

SOUZA, A.N.; OLIVEIRA, A.D. de; SCOLFORO, J.R.S.; REZENDE, J.L.P. de; MELLO, J.M. de. Viabilidade econômica de um sistema agroflorestal. Cerne, v.13, p.96-106, 2007.

TSUKAMOTO FILHO, A.A. Fixação do carbono em um sistema agroflorestal com eucalipto na região do Cerrado em Minas Gerais. 2003. 98p. Tese (Doutorado) - Universidade Federal de Viçosa, Viçosa.

UDAWATTA, R.P.; GARRETT, H.E.; KALLENBACH, R.L. Agroforestry and grass buffer effects on water quality in grazed pastures. Agroforestry Systems, v.79, p.81-87, 2010.

VALE, R.S. do; COUTO, L.; SILVA, M.L. da; GARCIA, R.; ALMEIDA, J.C. de C.; LANI, J.L. Análise da viabilidade econômica de um sistema silvipastoril com eucalipto para a Zona da Mata de Minas Gerais. Agrossilvicultura, v.1, p.107-120, 2004.

ZONETE, F.M.; RODRIGUEZ, L.C.E.; PACKALÉN, P. Estimação de parâmetros biométricos de plantios clonais de eucalipto no sul da Bahia: uma aplicação da tecnologia laser aerotransportada. Scientia Forestalis, v.38, p.225-235, 2010.

Recebido em 18 de agosto de 2011 e aprovado em 23 de janeiro de 2012

Pesq. agropec. bras., Brasília, v.47, n.2, p.253-260, fev. 2012 